\title{
Schwanoma retroperitoneal benigno, exéresis por laparoscopia
}

\author{
Mir Maresma C, Raventós CX, Celma A, Bestard Vallejo J, Orsola De los Santos A, \\ Morote Robles J.
}

Servicio de Urología. Hospital Vall d’Hebrón. Universitat Autónoma de Barcelona. Barcelona.

Actas Urol Esp. 2008;32(4):455-457

\section{RESUMEN}

SCHWANOMA RETROPERITONEAL BENIGNO, EXÉRESIS POR LAPAROSCOPIA

Los Schwanomas son tumores raramente localizados en el retroperitoneo, ya que habitualmente se encuentran en nervios craneales o periféricos. Raramente se diagnostican preoperatoriamente ya que ninguna de las técnicas de imagen es capaz de determinarlos con certeza. La RMN parece la prueba de elección. La exéresis quirúrgica completa es el tratamiento estándar. El caso que presentamos corresponde a un schwanoma retroperitoneal benigno como hallazgo a partir de dolor lumbar, en el describimos por primera vez la resección laparoscópica de este tipo de tumores.

Palabras clave: Schwanoma retroperitoneal. Tumores retroperitoneo laparoscopia.

\section{ABSTRACT}

\section{BENIGNE RETROPERITONEAL SCHWANNOMA, LAPAROSCOPIC RESSECTION}

Schwannomas are tumors rarely localized in the retroperitoneum, generally appear in craneal as well as periferic nerves. Seldom cases the diagnosis is preoperatively made just because imaging is very poor in this field. MRI is proven to be the diagnostic method. Radical surgical ressection is standarized treatment. We document a case of a benign retroperitoneal schwannoma where we explain the laparoscopic ressection of this kind of tumors for first time.

Keywords: Retroperitoneal schwannoma. Retroperitoneal tumors. Laparoscopics.

$\mathrm{L}$ os tumores originados en la vaina nerviosa son un subtipo de neoplasias de tejidos blandos que incluyen los schwanomas benignos y malignos ${ }^{1}$. Los Schwanomas se dan habitualmente en nervios craneales y periféricos, sólo en un 3\% de los casos se presentan en el retroperitoneo, donde presentan una mayor tendencia a la degeneración espontánea y a la hemorragia. Los Schwannomas se asocian con frecuencia con la enfermedad de von Recklinghausen en 5-18\% de los casos. Se presenta en edades comprendidas entre 20 y 50 años, únicamente un 5\% debutan en adolescencia.

\section{CASO CLÍNICO}

Presentamos el caso de una mujer de 52 años con antecedentes patológicos de hipertensión controlada con tratamiento dietético y apendicetomía en la infancia, sin hábitos tóxicos. A raíz de dolor lumbar izquierdo de meses de evolución se solicita ecografía abdominal que informa de riñones de ecoestructura normal, sin apreciar dilatación de vías excretoras ni imágenes sugestivas de litiasis. En la situación postero-medial al riñón izquierdo se observa una masa sólida no pulsátil de 74 x $62 \mathrm{~mm}$ con aparente pedículo que parece extenderse hacia seno renal sugestivo de tumor renal. A la exploración física no se objetivan tumoraciones móviles en fosa lumbar.

Se completa estudio con TAC abdominal que informa de masa encapsulada de coeficiente de atenuación semisólido en zona paraórtica izquierda con pequeñas calcificaciones en su interior que produce compresión extrínseca sobre la mencionada vena renal y el riñón izquierdo con aparente interfase con el mismo. (Fig. 1).

Se decide practicar exéresis de masa retroperitoneal izquierda paraórtica por laparoscopia. 


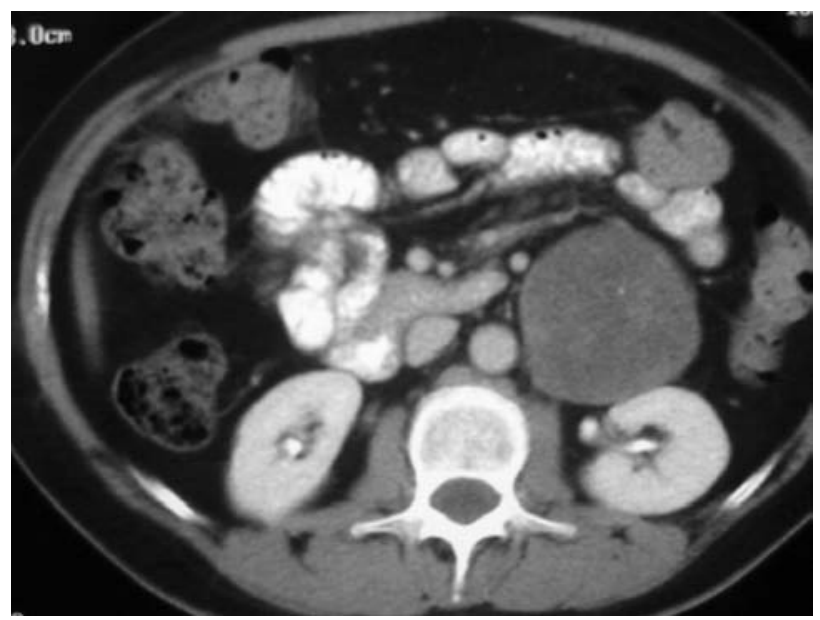

FIGURA 1. TAC que muestra masa de unos $8 \mathrm{~cm}$ de diámetro en retroperitoneo izquierdo, encapsulada, sin calcificaciones ni degeneración quistica.

En la cirugía encontramos una gran masa encapsulada, bien delimitada, que no infiltraba músculo psoas (Fig. 2). El postoperatorio cursó sin complicaciones y fue dada de alta al tercer día.

El análisis anatomopatológico definitivo de la pieza reveló schwanoma focalmente hialinizado y sin atípias, de 7,2 x 5,2 x 5,5 cm y $130 \mathrm{~g}$ de peso. La tumoración se mostraba encapsulada con superficie lisa blanco-grisácea y consistencia firme con algunas áreas hemorrágicas. Se identifica además un nódulo blanquecino de $5 \mathrm{~mm}$ de diámetro de consistencia más dura (Figs. 3 y 4).

Seguimiento postoperatorio en nuestras consultas externas con buena recuperación, presentándose libre de enfermedad a los 4 meses de seguimiento.

\section{COMENTARIOS}

El Schwanoma es un diagnóstico infrecuente para el urólogo; de hecho, en una revisión realizada a partir de 303 schwanomas, solamente $2(0,7 \%)$ se localizaban en retroperotineo ${ }^{2}$.

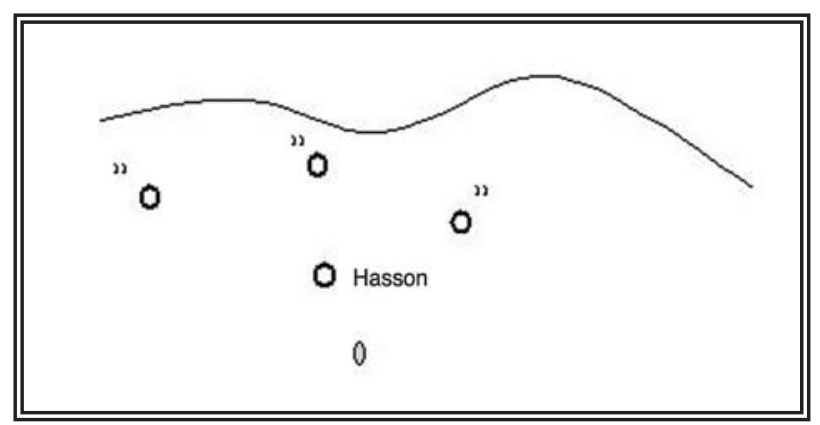

FIGURA 2. Esquema de la colocación de los trócares.

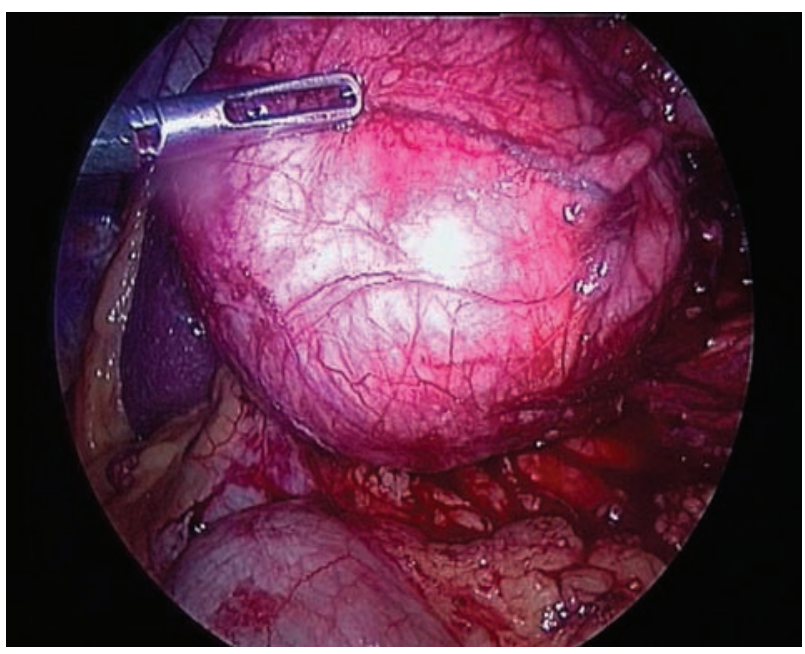

FIGURA 3. Imagen macroscópica de la tumoración.

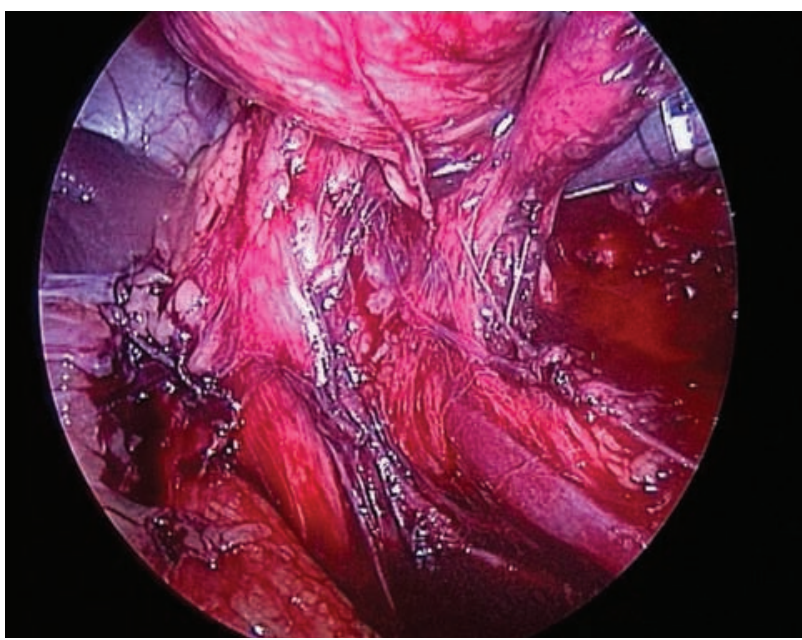

FIGURA 4. Imagen macroscópica del hilio renal.

Son tumores que acostumbran a tener gran tamaño al momento del diagnóstico (algunas series describen tamaños entre 8 y $20 \mathrm{~cm})^{4}$ debido probablemente a la flexibilidad y laxitud del espacio retroperitoneal que determina además el hecho que se diagnostiquen como hallazgos casuales dado que raramente dan alguna sintomatología. Los síntomas, si existen, son inespecíficos. Los síntomas neurológicos son raros como muestra una serie de 7 pacientes en los que sólo 2 presentaban síntomas de los cuales ninguno era neurológico ${ }^{3}$.

Se trata de tumores que se caracterizan además por tener un crecimiento lento que explica el porque la sintomatología es tan poca.

Macroscópicamente, los schwanomas son tumores únicos, bien delimitados, encapsulados que afectan fibras nerviosas periféricas. Debido a su gran tamaño 
tienen tendencia a presentar cambios secundarios como degeneración quística, calcificaciones, hemorragia, hialinización. Una vez muestran este tipo de degeneraciones se denominan "schwanomas ancianos"5.

Microscópicamente, estos tumores están formados por células de Schwann con regiones de alta y baja celularidad llamadas Antoni A y Antoni B, respectivamente, con tinciones altamente positivas para la proteína $\mathrm{S} 100$.

Las pruebas de imagen, generalmente ecografía y TAC nos ayudan a determinar el tamaño, localización, invasión o implicación de órganos vecinos; pero ninguna de ellas es patognomónica para este tumor ${ }^{3}$. La ecografía es útil y barata para detectarlos por la presencia de masa quística con áreas semisólidas en el retroperitoneo ya que la mayoría de tumores del retroperitoneo no son quísticos. En una serie de 133 casos de schwanomas retroperitoneales, el 66\% mostraban degeneración quística ${ }^{6}$. En una serie de 7 casos presentada recientemente, $3(42,9 \%)$ tumores presentaban degeneración quística ${ }^{3}$.

La TAC habitualmente describe masas redondas o ovaladas, bien delimitadas con calcificaciones puntiformes y degeneración quística ${ }^{7}$.

El diagnóstico diferencial de tumores sólidos retroperitoneales incluye tumores retroperitoneales como neurofibromas, paragangliomas, feocromocitomas, liposarcomas, histocitomas fibrosos malignos $^{8}$. Estos tumores pueden también simular neoplasias adrenales como muestra la serie de Brian KP donde 3 de los 7 casos presentados estaban diagnosticados preoperatoriamente de neoplasia adrenal. De hecho, ya Beherend $\mathrm{M}$ et al., mostraban un caso similar que únicamente pudo ser diagnosticado en la resección quirúrgica ${ }^{9-10}$.

La prueba de imagen que mejor nos ayuda a caracterizar este tipo de tumores es la resonancia magnética nuclear ya que nos permite una mejor visualización del origen, arquitectura vascular y implicación de estructuras vecinas. Típicamente, estos tumores se presentan isointensos en T1 y hiperintensos en T2. Pero, como siempre en estos casos, esto no es característica única para estos tumores y es únicamente una parte más del estudio ${ }^{11}$.

La TAC con PAAF es poco fiable, ocasionalmente puede ser útil si se obtienen muchas células de Schwann en la muestra. Presenta el inconveniente de la diseminación tumoral por siembra de células cancerosas, por lo que muchos autores la desaconsejan $^{4}$.
El tratamiento ideal para estos tumores es la resección completa. Existe controversia, ya que algunos autores defienden la resección completa con márgenes amplios incluyendo tejidos vecinos ${ }^{3-4}$ dado que el riesgo de malignidad no puede excluirse preoperatoriamente; otros propugnan la simple enucleación al tratarse de un tumor benigno ${ }^{12}$.

En conclusión, los tumores retroperitoneales son tumores raros con diagnóstico preoperatorio difícil debido a sus características radiológicas poco específicas. La RMN parece ser la prueba de elección en el diagnóstico. La TAC con PAAF parece relegada a un segundo plano. El tratamiento debe incluir la resección completa con márgenes negativos.

\section{REFERENCIAS}

1. Gubbay AD, Moschilla G, Gray BN, Thompson I. Retroperitoneal schwannoma: a case series and review. Aust N Z J Surg. 1995; 65(3):197-200.

2. Das Gupta TK, Brasfield RD, Strong EW, Hajdu SI. Benign solitary Schwannomas (neurilemomas). Cancer. 1969;24(2):355366.

3. Goh BK, Tan YM, Chung YF, Chow PK, Ooi LL, Wong WK. Retroperitoneal schwannoma. Am J Surg. 2006;192(1):14-18.

4. Daneshmand S, Youssefzadeh D, Chamie K, Boswell W, Wu N, Stein JP, et al. Benign retroperitoneal schwannoma: a case series and review of the literature. Urology. 2003;62(6):993-997. Review.

5. Bezzi M, Orsi F, Rossi P. Nonencapsulated ancient schwannoma of the renal sinus. AJR Am J Roentgenol. 1996;166(6): 1498.

6. Takatera H, Takiuchi H, Namiki M, Takaha M, Ohnishi S, Sonoda T. Retroperitoneal schwannoma. Urology. 1986;28(6): 529-531.

7. Kinoshita T, Naganuma H, Ishii K, Itoh H. CT features of retroperitoneal neurilemmoma. Eur J Radiol. 1998;27(1):67-71.

8. Kransdorg MJ. Benign soft-tissue tumors in a large referral population: distribution of specific diagnoses by age, sex, and location. AJR Am J Roentgenol. 1995;164(2):395-402.

9. Behrend M, Kaaden S, Von Wasielewski R, Frericks B. Benign retroperitoneal schwannoma mimicking an adrenal mass. Surg Laparosc Endosc Percutan Tech. 2003;13(2):133-138.

10. Maeshima S, Nakamura H, Nishikawa M, Murakami T, Mitani T, Marukawa T, et al. Retroperitoneal schwannomas simulating adrenal tumors. Clin Imaging. 1992;16(2):121-124.

11. Hayasaka K, Tanaka Y, Soeda S. MR findings in primary retroperitoneal schwannoma. Acta Radiol. 1999;40(1):78-82.

12. Miller PL, Tessler A, Alexander S, Pinck BD. Retroperitoneal neurilemmoma. Urology. 1978;11(6):619-623.

Correspondencia autora: Dra. C. Mir Maresma

Servicio de Urología. Hospital Vall d'Hebrón.

Passeig de la Vall D’Hebrón, 119-129 - 08035 Barcelona

Tel.: 932746000

E-mail autora: mirmare@yahoo.es

Información artículo: Nota Clínica

Trabajo recibido: octubre 2006

Trabajo aceptado: noviembre 2006 